\title{
Clinical use of trastuzumab combined with different chemotherapy regimens in multi-line treatment of advanced human epidermal growth factor receptor 2-positive gastric cancer: A case report
}

\author{
ZHE-LING CHEN, ANDI ZHAO, PAN LI, MI ZHANG, JIAO YANG, LINGXIAO ZHANG, \\ XIAOAI ZHAO, JIN YANG and LE WANG \\ Department of Medical Oncology, The First Affiliated Hospital of Xi'an Jiaotong University, \\ Xi'an, Shaanxi 710061, P.R. China
}

Received November 8, 2017; Accepted June 14, 2018

DOI: $10.3892 / \mathrm{ol} .2018 .9212$

\begin{abstract}
It is generally acknowledged that gastric cancer requires comprehensive treatment approaches to be adopted. For patients with human epidermal growth factor receptor-2 (HER2)-overexpressing gastric cancer, targeting HER2 with trastuzumab in first-line therapy combined with standard chemotherapy significantly improves the prognosis. However, there is a lack of international guidance for second-line treatment if a patient experiences disease progression. There is also no accepted conclusion regarding the efficiency of cross-line therapy with trastuzumab. The present study reports the case of a 55-year-old male with gastric cancer who underwent radical gastrectomy. Immunohistochemistry indicated that samples were EGFR(+) and HER-2(3+), with Ki-67 (20\%). From abdominal computed tomography scanning and contrast-enhanced ultrasound following surgery, hepatic metastasis was identified and the patient was administered microwave thermocoagulation therapy. Since December 2012, the patient received multi-line chemotherapy regimens as follows: i) Oxaliplatin, tegafur/gimeracil/oteracil and trastuzumab; ii) paclitaxel liposome and S-1 plus trastuzumab; iii) apatinib; iv) epirubicin/oxaliplatin/xeloda; and v) irinotecan plus trastuzumab. During the course of therapy, the trastuzumab served an important function in multi-line therapy and the patient benefited from the combined therapy. The application of trastuzumab in the multi-line treatment of
\end{abstract}

Correspondence to: Professor Jin Yang or Dr Le Wang, Department of Medical Oncology, The First Affiliated Hospital of Xi'an Jiaotong University, 277 West Yanta Road, Xi'an, Shaanxi 710061, P.R. China E-mail: 1473106133@qq.com

E-mail: lele19810104@sina.com

Key words: trastuzumab, multi-line treatment, gastric cancer, human epidermal growth factor receptor 2-positive a patient with HER2-positive advanced gastric cancer may be worthy of investigation for use in the clinic.

\section{Introduction}

In the past 20 years, gastric cancer has remained one of the most common types of malignant tumor and is a common cause of cancer-associated mortality worldwide (1). Data from the National Central Cancer Registry in 2013 indicated that the incidence of gastric cancer in China was $0.042 \%$, which was second only to lung cancer (2). For those patients suffering with unresectable tumors or recurrent disease, it is generally acknowledged that comprehensive treatment with systemic medication, including chemotherapy, targeted drugs and immunotherapy, should be adopted $(3,4)$.

The overexpression rates of human epidermal growth factor receptor-2 (HER2) in gastric cancer have been demonstrated to range between 7.3 and $22.1 \%(5,6)$. A retrospective study demonstrated that HER2 expression is associated with aggressive biological behavior (7). HER2 expression is an indicative factor of the efficacy of trastuzumab treatment. In the Trastuzumab for Gastric Cancer (ToGA) study, targeting HER 2 with trastuzumab in first-line therapy combined with standard chemotherapy significantly improved the overall survival of patients compared with standard chemotherapy alone (16.0 vs. 11.8 months) (8). Results from certain phase II clinical trials revealed longer overall survival and reduced side effects resulting from trastuzumab in combination with other chemotherapy regimens $(9,10)$. However, the present literature is incomplete and lacks data from randomized controlled clinical trials. Additionally, there are no guidelines to aid physicians in reaching a decision for patients who require third-line or further chemotherapy. Only apatinib is approved for use as a third-line treatment of refractory gastric cancer in China (11). Other studies on irinotecan or docetaxel are limited by small sample sizes (12-14). Thus, there is currently a lack of an international standard for patients following second-line treatment.

The present study reports the case of a patient with gastric cancer who received multi-line chemotherapy, including 
treatment with trastuzumab. The course of treatment is presented in Fig. 1.

\section{Case report}

A 55-year-old male patient visited the First Affiliated Hospital of Xi'an Jiaotong University (Xi'an, China) in December 2012 with the diagnosis of gastric tumor from Xijing Hospital, the Fourth Military Medical University. The patient underwent radical gastrectomy in November 2011. Surgical specimens were fixed with $4 \%$ neutral buffered formaldehyde for $8 \mathrm{~h}$ at room temperature. The postoperative pathological results revealed moderately and poorly differentiated adenocarcinoma. Metastatic disease was observed in nodes at the lesser curvature (1/1) and greater curvature $(5 / 13)$ of the stomach. Immunohistochemistry indicated that the samples were epidermal growth factor receptor (EGFR)(+) and HER-2(3+), with Ki-67 (a cellular marker for proliferation, 20\%) (Fig. 2). The immunohistochemical detection process was based on to the 2011 version of HER2 detection guidelines for gastric cancer $(15,16)$. Envision method was used for HER2 staining. Surgical specimens were the selected samples for HER2 staining which were fixed with $4 \%$ neutral buffered formaldehyde for $8 \mathrm{~h}$ at room temperature, then embedded in paraffin wax. Slice thickness was between 3 and $5 \mu \mathrm{m}$. Cooking boiling and heating methods were used for antigen retrieval (dewaxed tissue section was placed into $0.01 \mathrm{~mol} / 1$ citrate buffer heated to $95^{\circ} \mathrm{C}$ for $15 \mathrm{~min}$; then keep warm for $10 \mathrm{~min}$; finally naturally cooled to room temperature). Then sample were incubated with $3 \% \mathrm{H}_{2} \mathrm{O}_{2}$ for $10 \mathrm{~min}$ at room temperature to block the interstitial source hyperparathyroid activity. Primary antibody (Anti-ERBB2; cat. no. ab134182) was obtained from Abcam (Cambridge, USA) and diluted into working fluids (1:500), which bound to the extracellular domain of human ErbB2. A total of $50 \mu \mathrm{l}$ primary antibody working fluids were added for $30 \mathrm{~min}$ at room temperature. DAB working fluid was added at room temperature for 5-30 min. Then the HER 2 expression was observed under a bright field of vision under a light microscope at a magnification $\mathrm{x} 400$. The HER2 scoring system was as follows: HER2 (-), no positive cells or membrane-positive cells $<10 \%$; HER2 (1+), $10 \%$ of cells have faint/inappreciable membrane staining or only partial cell membrane staining; HER2 (2+), >10\% of cells have weak, or medium-intensity, complete U-type film or II-type coloring; HER2 (3+), >10\% of cells have medium or strong intact U-type membranes or II-type coloring.

From abdominal computed tomography (CT) and contrast-enhanced ultrasound scanning following surgery, hepatic metastasis was identified. The patient then received microwave thermocoagulation therapy. Between December 2012 and June 2013, the patient received chemotherapy with the SOX regimen for 8 cycles [oxaliplatin (L-OHP), $130 \mathrm{mg} / \mathrm{m}^{2}$ on day 1, and tegafur/gimeracil/oteracil (S-1) $50 \mathrm{mg}$ twice daily on days $1-14$, every 3 weeks]. During this period, combined treatment with trastuzumab was performed 12 times $(8 \mathrm{mg} / \mathrm{kg}$ first dose and $6 \mathrm{mg} / \mathrm{kg}$ followed on day 0 , every 3 weeks) and the disease remained stable. Trastuzumab combined with S-1 was used as the maintenance regimen of the first-line therapy, which lasted 12 cycles.

In November 2015, the patient returned and was reexamined. At this time, $\mathrm{CT}$ examination revealed mediastinal and retro-peritoneal lymphadenectasis, which indicated disease progression. Considering the benefit from the use of trastuzumab in first-line chemotherapy (disease control was maintained for 26 months), a second-line regimen of 6 cycles of paclitaxel liposome (L-PTX, $175 \mathrm{mg} / \mathrm{m}^{2}$ on day 1) and S-1 (50 mg twice daily on days 1-14) together with trastuzumab ( $8 \mathrm{mg} / \mathrm{kg}$ first dose and $6 \mathrm{mg} / \mathrm{kg}$ followed on day 0 ) was selected. A CT scan in January 2016 revealed that the mediastinal and retro-peritoneal lymphadenectasis had decreased in size (Fig. 3). Furthermore, the tumor marker levels from the serum sample (carcinoembryonic antigen and cancer antigen) were markedly decreased (Fig. 4). In March 2016, 10-MV radiotherapy (tumor absorption dose $50 \mathrm{~Gy} / 25$ fraction) targeting abdominal lymph node lesions was performed. Between March 2016 and August 2016, maintenance treatment of trastuzumab combined with S-1 was administered. The disease was kept stable and the tumor markers exhibited little fluctuation until a new pulmonary nodule was observed during a CT scan in August 2016. In the subsequent 5 months, the targeted drug was changed to apatinib (250 mg twice daily, from August 2016 to December 2016) due to newly observed pulmonary lesions. In December 2016, a further CT scan revealed disease progression resulting from the pulmonary lesions (Fig. 5).

There is no widely-accepted agreement on the fourth-line therapy that should be used to treat advanced gastric tumors. In the present study, the EOX chemotherapy regimen was utilized for the patient (epirubicin, $50 \mathrm{mg} / \mathrm{m}^{2}$ on day 1 ; L-OHP, $130 \mathrm{mg} / \mathrm{m}^{2}$ on day 1 ; xeloda $1,000 \mathrm{mg} / \mathrm{m}^{2}$ on days $1-14$, every 3 weeks) for 5 cycles. The tumor marker levels declined after 2 cycles and the disease was controlled stably for 6 months. However, a CT scan in May 2017 revealed multiple new lesions in the lung (Fig. 5).

Up to this point, the patient had been treated with a combined chemotherapy regimen with a HER2-targeting strategy using multiple lines of therapy. When faced with the choice of the fifth-line therapy, trastuzumab together with irinotecan $\left(120 \mathrm{mg} / \mathrm{m}^{2}\right.$, days 1 and 8$)$ was selected. The lung lesion was reduced in size and the tumor marker level was markedly decreased (Fig. 5). The left ventricular ejection fraction (LVEF), the most considerable toxic reaction of trastuzumab, was kept stable during the therapy period (range, $62-74 \%$ ) and the maximum decrease in LVEF was $<16 \%$. The hematological toxicity was primarily leucopenia. Currently, the patient remains under treatment in a stable condition.

\section{Discussion}

The present study reports the case of a patient with HER2-overexpressing metastatic gastric cancer experiencing survival benefits from multi-line chemotherapy combined with trastuzumab. At the time of writing, fifth-line chemotherapy has been administered to the patient and the treatment with trastuzumab has been utilized three times.

Previously, a subgroup analysis of ToGA demonstrated that patients with immunohistochemistry staining scores of $3+$ or $2+$ together with positive fluorescence in situ hybridization results could experience survival benefits from trastuzumab combined with chemotherapy (17). The comparison of the median overall survival (mOS) was more predominant 


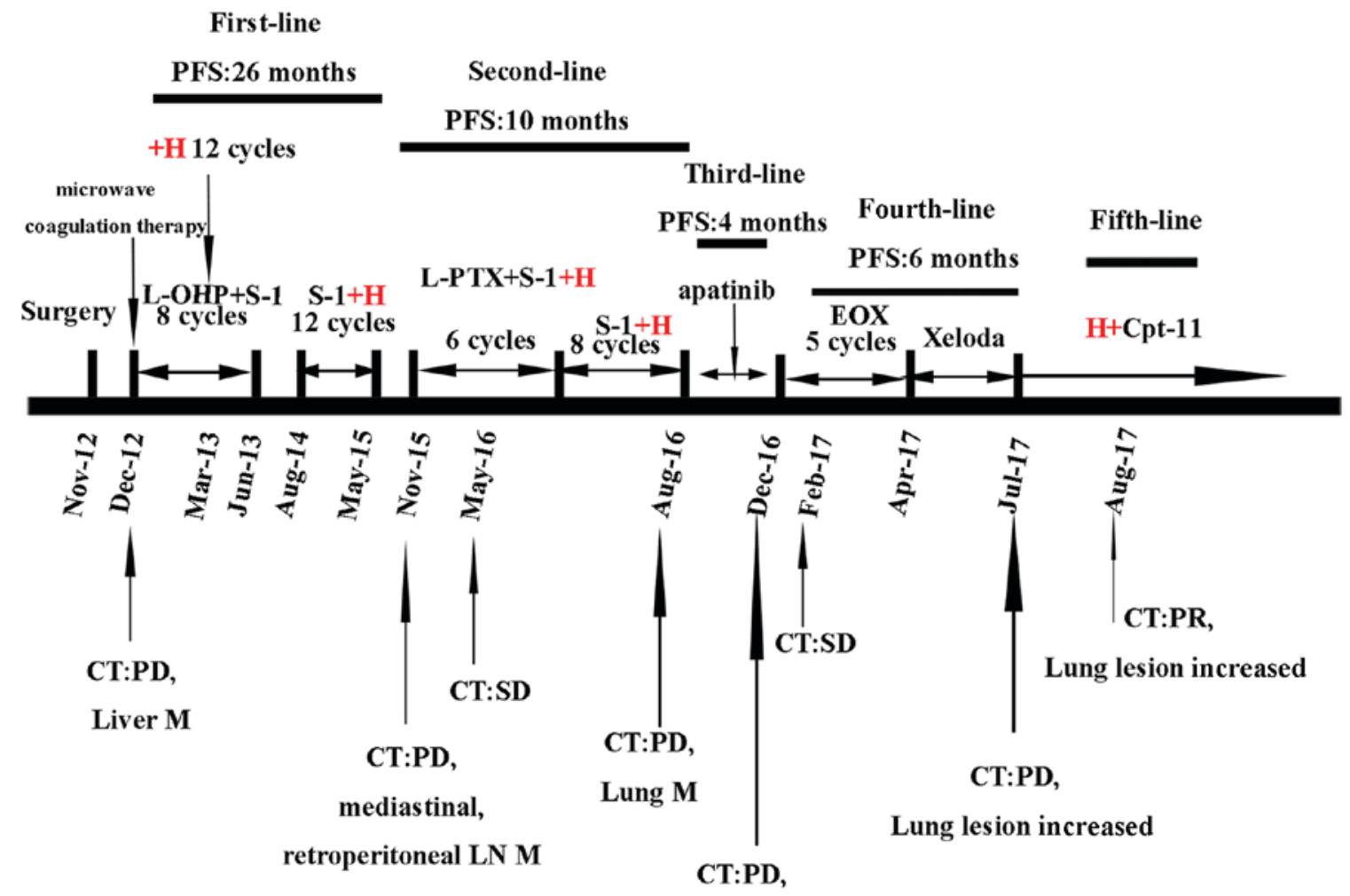

Lung lesion increased

Figure 1. Multi-line treatment schedule. The patient received multi-line chemotherapy combined with trastuzumab following disease progression. CT, computed tomography; PD, progressive disease; SD, stable disease; PR, partial response; PFS, progression-free survival; M, metastasis; LN, lymph node; H, trastuzumab; L-OHP, oxaliplatin; S-1, tegafur gimeracil oteracil potassium; L-PTX, paclitaxel liposome; Cpt-11, irinotecan; EOX, epirubicin/oxaliplatin/xeloda; XP, xeloda/cisplatin; CEA, carcino-embryonic antigen; AFP, $\alpha$ fetoprotein.

A

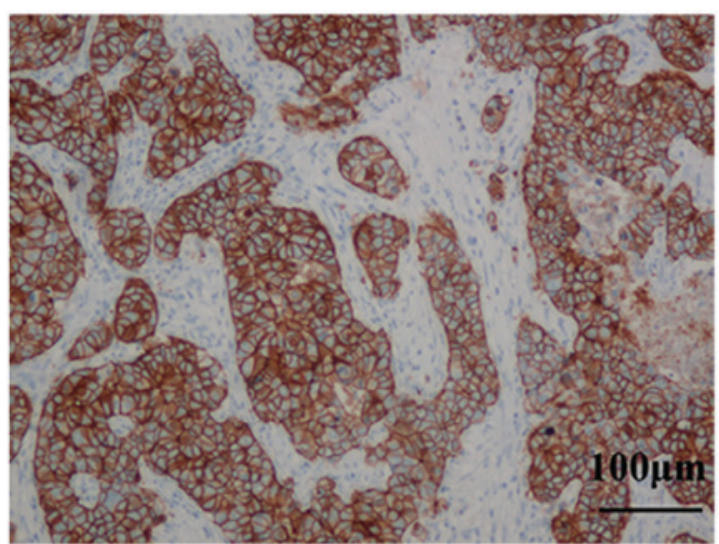

B

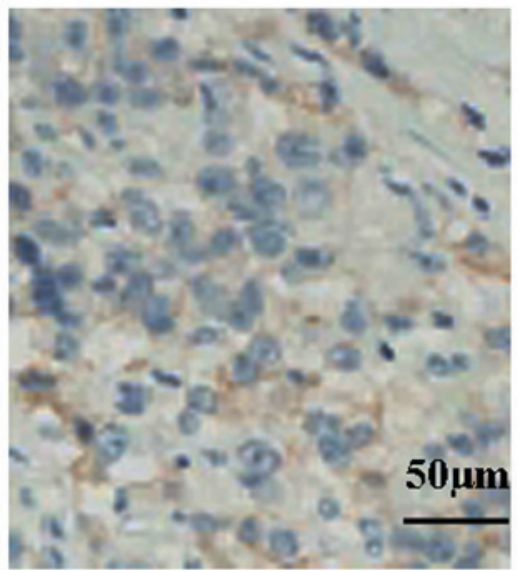

Figure 2. Expression of HER2 as assessed by immunohistochemistry. (A) HER2 was overexpressed in the cancer tissues. Scale, $100 \mu$ m. (B) The samples not incubated with antibody were used as the negative control. Scale, $50 \mu \mathrm{m}$. HER2, human epidermal growth factor receptor.

(16.0 vs. 11.8 months) than that in the total cohort $(13.8$ vs. 11.1 months) (8). Therefore, the combination of trastuzumab with cisplatin and fluorouracil (FU) or capecitabine (CAP) is considered the standard first-line chemotherapeutic regimen of advanced HER2-positive gastric cancer. Certain other phase II clinical trials have evaluated the safety and efficiency of trastuzumab combined with other chemotherapy regimens, including CAP plus L-OHP (9), S-1 plus cisplatin $(10,18)$ and CAP plus L-OHP (19). Although evidence suggests that a platinum-based drug combination is more likely to improve the survival of gastric cancer patients, the SOX plus trastuzumab regimen was selected in favor of $5 \mathrm{FU}$ or capecitabin plus CDDP as the first-line therapy due to the age and physical condition of the patient described in the present study. Compared with cisplatin and carboplatin, L-OHP has a reduced toxic effect. In clinical trials, the L-OHP contained regimens, including EOF, EOX and SOX, which demonstrated good efficiency and safety $(20,21)$. In 
A
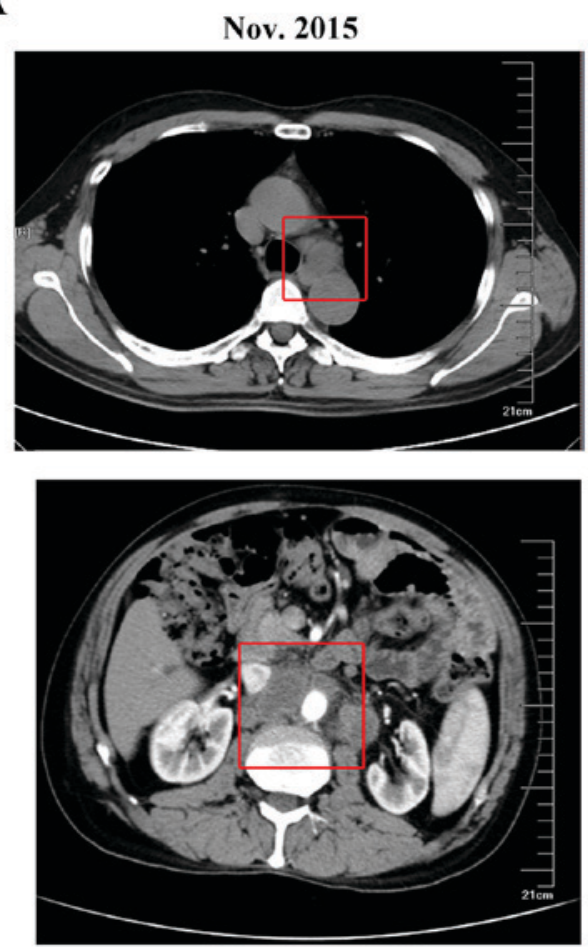

B
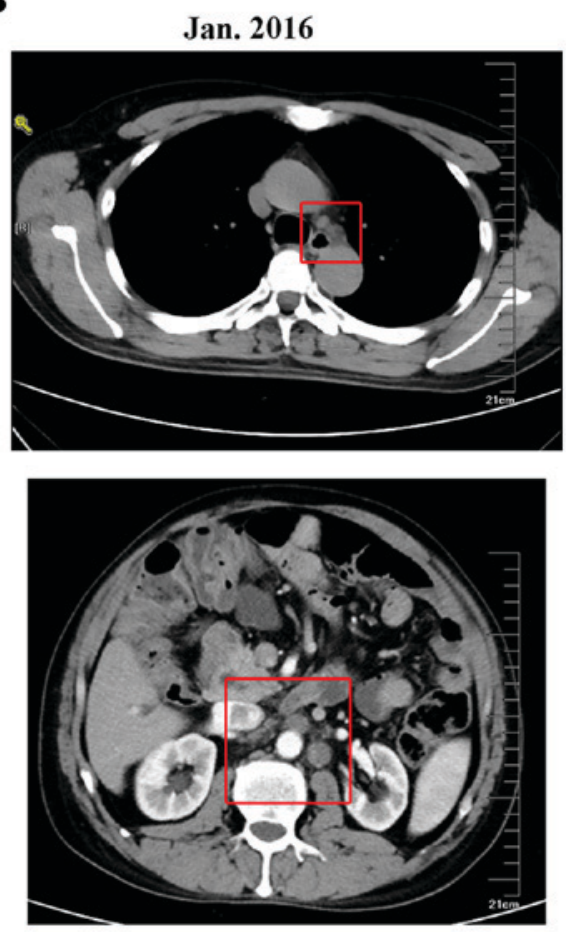

Figure 3. CT scans comparing the mediastinal/retroperitoneal lymphadenectasis prior to and following the second-line chemotherapy regimen combined with trastuzumab. (A) CT in November 2015 revealed mediastinal (upper) and retroperitoneal (lower) lymphadenectasis. (B) CT in January 2016 demonstrated that the mediastinal (upper) and retroperitoneal (lower) lymphadenectasis was reduced in size following second-line chemotherapy combined with trastuzumab. CT, computed tomography.

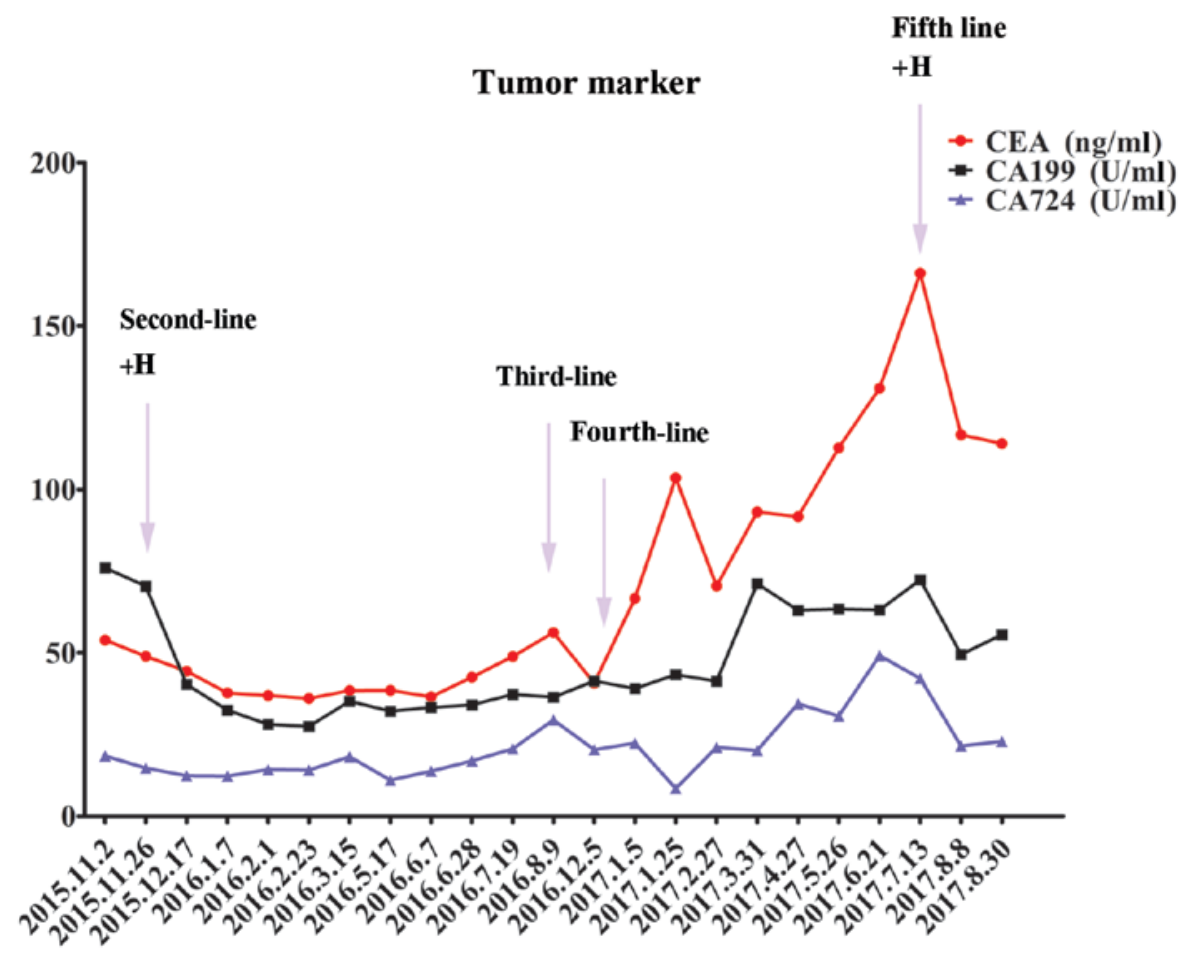

Figure 4. Tumor marker levels. The change in CEA (ng/ml), CA19-9 (U/ml) and CA72-4 (U/ml) levels over time. H, trastuzumab; CEA, carcinoembryonic antigen; $\mathrm{CA}$, cancer antigen.

the present case, first-line use of trastuzumab with the SOX chemotherapy regimen achieved successful stabilization of the disease for $>2$ years.
For those patients who experience progression following first-line treatments, large randomized clinical trials are lacking, and no consistent treatment recommendation is 
Table I. Summary of clinical trials containing second-line chemotherapy.

Survival time, months

\begin{tabular}{|c|c|c|c|c|c|}
\hline First author & Year & Regimen & PFS & OS & (Refs.) \\
\hline Thuss-Patience et al & 2011 & Irinotecan & l & 4.0 & (27) \\
\hline Kang et al & 2012 & $\begin{array}{l}\text { Irinotecan, } \\
\text { Docetaxel }\end{array}$ & l & $\begin{array}{l}6.5 \\
5.2\end{array}$ & (12) \\
\hline Meads et al & 2014 & Docetaxel & 1 & 5.2 & (23) \\
\hline Hironaka et al & 2013 & $\begin{array}{l}\text { Paclitaxel } \\
\text { Irinotecan }\end{array}$ & $\begin{array}{l}3.6 \\
2.3\end{array}$ & $\begin{array}{l}9.5 \\
8.4\end{array}$ & (22) \\
\hline Sym et al & 2013 & $\begin{array}{c}\text { Irinotecan } \\
\text { Irinotecan plus 5-FU/leucovorin }\end{array}$ & $\begin{array}{l}2.2 \\
3.0\end{array}$ & $\begin{array}{l}5.8 \\
6.7\end{array}$ & (28) \\
\hline Maruta et al & 2007 & $\begin{array}{c}\text { Docetaxel } \\
\text { Docetaxel plus 5-FU }\end{array}$ & I & $\begin{array}{l}4.0 \\
7.6\end{array}$ & (29) \\
\hline
\end{tabular}

PFS, progression-free survival; OS, overall survival; 5-FU, f-fluorouracil.

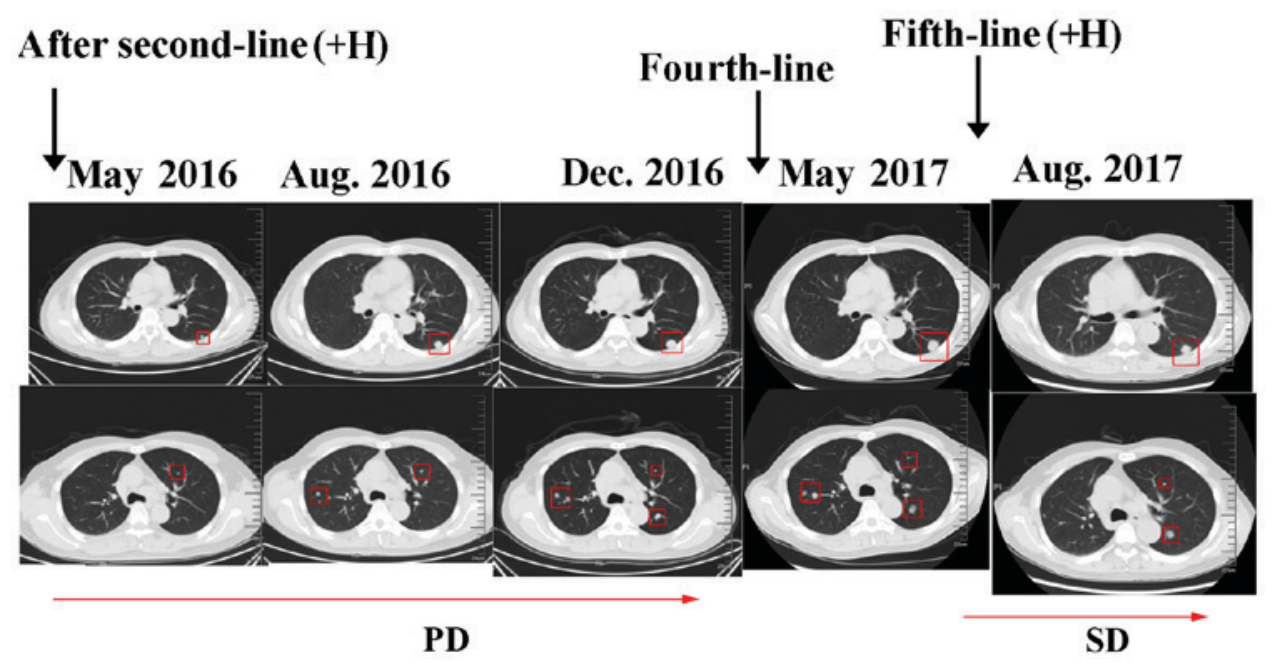

Figure 5. CT scans. CT scans demonstrated the change in the lung metastasis lesions during multi-line chemotherapy regimens combined with trastuzumab. The disease exhibited good response to trastuzumab plus chemotherapy even in fifth-line treatment. H, trastuzumab; PD, progressive disease; SD, stable disease; CT, computed tomography.

available. Based on the results of the COUGAR-02 (22) and WJOG 4007 (23) studies, second-line chemotherapeutic drugs for advanced gastric cancer patients are predominantly taxanes or irinotecan. In the National Comprehensive Cancer Network Clinical Practice Guidelines for Gastric Cancer, it is recommended to use trastuzumab for posterior line therapy in patients with HER2-positive advanced gastric cancer who do not use trastuzumab in first-line therapy (24). The JFMC45-1102 phase II clinical trial demonstrated the satisfactory survival benefit of a weekly dose of paclitaxel combined with trastuzumab, including progression-free survival (PFS) of 5.1 months) and mOS of 16.8 months, in patients with HER2-positive cancer who did not receive trastuzumab treatment previously (25). These data all support the use of trastuzumab in second-line therapy. However, the prospective data about cross-line application of trastuzumab treatment is lacking. Whether trastuzumab may be used effectively alongside another chemotherapy regimen following disease progression is unknown. Based on the good reactivity of the present patient to trastuzumab treatment in first-line therapy, trastuzumab plus Tegafur Gimeracil Oteracil Potassium (S-1) and paclitaxel liposome was attempted in second-line therapy (26). The disease remained stable for 10 months. Table summarizes the regimens and survival duration of patients in clinical trials with second-line treatment reported in the literature $(12,22,23,27-29)$. The PFS ranged from 2.2 to 3.6 months and the OS ranged from 4.0 to 9.5 months (Table I). The survival time of the present patient exceeded the median survival time presented in those studies that contained only chemotherapy as the second-line therapy. Thus, we hypothesize that the targeted agent may bring benefits to patients with advanced disease even subsequent to first-line therapy. Other drugs targeting HER2, including lapatinib, trastuzumab emtansine (T-DM1) and anti-angiogenic drugs, such as ramucirumab 
and bevacizumab, are also alternative treatments in the case of first-line treatment failure $(30,31)$. The REGARD (32) and RAINBOW (33) trials analyzed the efficiency of ramucirumab in the second-line treatment of HER2-amplified advanced gastric cancer. No matter the single-agent use or combination with paclitaxel, ramucirumab significantly improved the mOS for nearly 2 months. Therefore, ramucirumab in combination with taxane drugs is approved by the Food and Drug Administration for the second-line treatment of advanced gastric cancer. This provides novel options for physicians to treat cancer, which will be of benefit to patients.

To date, there has been a lack of randomized controlled clinical trials to support third-line treatment, but certain retrospective studies have identified that patients receiving multi-line treatment have experienced improved survival times $(34,35)$. A phase III trial verified that apatinib treatment significantly improved OS (6.5 vs. 4.7 months) and PFS (2.6 vs. 1.8 months) times following two or more prior lines of failed chemotherapy (36). A number of associated studies regarding immune checkpoint inhibitors, including pembrolizumab and nivolumab, have been performed $(37,38)$. In the present study, when taking the previous treatment and the condition of the patient into account, it was decided that continuing therapy was possible and apatinib was selected as the third-line therapy, which lasted for 4 months and was then changed to the EOX regimen. The fourth-line therapy controlled the disease for 6 months. The disease then progressed further and the regimen was changed to trastuzumab plus irinotecan. The use of trastuzumab combined with different chemotherapy regimens in cross-line treatment has contributed to the survival benefit of this patient and demonstrated the efficiency of trastuzumab with good safety.

The present study also has a number of limitations. Firstly, although the expression of HER2 was determined using immunohistochemistry, the absence of corroborating western blotting data and the lack of immunohistochemistry on non-cancerous tissue samples are limitations of this study. Secondly, the effect of this cross-line application of trastuzumab should be evaluated in numerous situations in the future, as opposed to in a single patient. Although the disease was controlled well, cardiac and hematological toxicities were also observed in this case. Therefore, the long-term safety and effectiveness of this therapy require evaluation in subsequent follow-ups.

In conclusion, the application of trastuzumab in multi-line treatment for a patient with HER2-positive advanced gastric cancer appears to have benefited the survival of the present patient, and may be of benefit to others in the future.

\section{Acknowledgements}

Not applicable.

\section{Funding}

No funding was received.

\section{Availability of data and materials}

The datasets generated and/or analyzed during the current study are available in the SEERdatabase (National Cancer Institute) repository, http://seer.cancer.gov/seerstat.

\section{Authors' contributions}

LZ and LW gave contributions to conception and design. LZ, PL, XZ and LW reviewed the literature and designed the article structure. JinY, AZ and MZ contributed to the acquisition and analysis of data. JY gave interpretation of data. ZC was a major contributor in writing the manuscript. XZ, JY and JinY revised and edited the manuscript critically for important intellectual content. ZC, LW, JY and JinY gave final approval of the version to be published.

\section{Ethics approval and consent to participate}

The present study was reviewed and approved by the First Affiliated Hospital of Xi'an Jiaotong University Ethics Committee.

\section{Patient consent for publication}

The patient provided written informed consent for publication.

\section{Competing interests}

The authors declare that they have no competing interests.

\section{References}

1. Ferlay J, Soerjomataram I, Dikshit R, Eser S, Mathers C, Rebelo M, Parkin DM, Forman D and Bray F: Cancer incidence and mortality worldwide: Sources, methods and major patterns in GLOBOCAN 2012. Int J Cancer 136: E359-E386, 2015.

2. Zheng R, Zeng H, Zhang $\mathrm{S}$ and Chen W: Estimates of cancer incidence and mortality in China, 2013. Chin J Cancer 36: 66, 2017.

3. Fukushima R: Recent progress in the treatment of advanced gastric cancer. Nihon Geka Gakkai Zasshi 113: 3, 2012 (In Japanese).

4. Xu JM: Comments on the seven clinical questions \& answers in Japanese gastric treatment guidelines of the 4th edition. Zhonghua Zhong Liu Za Zhi (Chinese J Oncol) 39: 236-238, 2017.

5. Van Cutsem E, Bang YJ, Feng-Yi F, Xu JM, Lee KW, Jiao SC, Chong JL, López-Sanchez RI, Price T, Gladkov O, et al: HER2 screening data from ToGA: Targeting HER2 in gastric and gastroesophageal junction cancer. Gastric Cancer 18: 476-484, 2015.

6. Cho J, Jeong J, Sung J, Sung CO, Kim KM, Park CK, Choi MG, Sohn TS, Bae JM and Kim S: A large cohort of consecutive patients confirmed frequent HER2 positivity in gastric carcinomas with advanced stages. Ann Surg Oncol 20 (Suppl 3): S477-S484, 2013.

7. Kim KC, Koh YW, Chang HM, Kim TH, Yook JH, Kim BS, Jang SJ and Park YS: Evaluation of HER2 protein expression in gastric carcinomas: Comparative analysis of 1,414 cases of whole-tissue sections and 595 cases of tissue microarrays. Ann Surg Oncol 18: 2833-2840, 2011.

8. Bang YJ, Van Cutsem E, Feyereislova A, Chung HC, Shen L, Sawaki A, Lordick F, Ohtsu A, Omuro Y, Satoh T, et al: Trastuzumab in combination with chemotherapy versus chemotherapy alone for treatment of HER2-positive advanced gastric or gastro-oesophageal junction cancer (ToGA): A phase 3, open-label, randomised controlled trial. Lancet 376: 687-697, 2010.

9. Gong J, Liu T, Fan Q, Bai L, Bi F, Qin S, Wang J, Xu N, Cheng Y, Bai Y, et al: Optimal regimen of trastuzumab in combination with oxaliplatin/capecitabine in first-line treatment of HER2-positive advanced gastric cancer (CGOG1001): A multicenter, phase II trial. BMC Cancer 16: 68, 2016.

10. Chua C, Tan IB, Yamada Y, Rha SY, Yong WP, Ong WS, Tham CK, Ng M, Tai DW, Iwasa S, et al: Phase II study of trastuzumab in combination with $\mathrm{S}-1$ and cisplatin in the first-line treatment of human epidermal growth factor receptor HER2-positive advanced gastric cancer. Cancer Chemother Pharmacol 76: 397-408, 2015. 
11. Brower V: Apatinib in treatment of refractory gastric cancer. Lancet Oncol 17: e137, 2016.

12. Kang JH, Lee SI, Lim DH, Park KW, Oh SY, Kwon HC, Hwang IG, Lee SC, Nam E, Shin DB, et al: Salvage chemotherapy for pretreated gastric cancer: A randomized phase III trial comparing chemotherapy plus best supportive care with best supportive care alone. J Clin Oncol 30: 1513-1518, 2012.

13. Kang EJ, Im SA, Oh DY, Han SW, Kim JS, Choi IS, Kim JW, Kim YJ, Kim JH, Kim TY, et al: Irinotecan combined with 5-fluorouracil and leucovorin third-line chemotherapy after failure of fluoropyrimidine, platinum, and taxane in gastric cancer: Treatment outcomes and a prognostic model to predict survival. Gastric Cancer 16: 581-589, 2013.

14. Tarazona N, Smyth EC, Peckit C, Chau I, Watkins D, Rao S, Starling $\mathrm{N}$ and Cunningham D: Efficacy and toxicity of salvage weekly paclitaxel chemotherapy in non-Asian patients with advanced oesophagogastric adenocarcinoma. Ther Adv Med Oncol 8: 104-112, 2016.

15. Guideline Recommendations for HER2 Detection in Gastric Cancer Group: Guidelines for HER2 detection in gastric cancer (2016). Zhonghua Bing Li Xue Za Zhi 45: 528-532, 2016.

16. Guideline Recommendations for HER2 Detection in Gastric Cancer Group: Guidelines for HER2 detection in gastric cancer. Zhonghua Bing Li Xue Za Zhi 40: 553-557, 2011.

17. Lei YY, Huang JY, Zhao QR, Jiang N, Xu HM, Wang ZN, Li HQ, Zhang SB and Sun Z: The clinicopathological parameters and prognostic significance of HER2 expression in gastric cancer patients: A meta-analysis of literature. World J Surg Oncol 15: 68, 2017.

18. Kurokawa Y, Sugimoto N, Miwa H, Tsuda M, Nishina S, Okuda H, Imamura H, Gamoh M, Sakai D, Shimokawa T, et al: Phase II study of trastuzumab in combination with $\mathrm{S}-1$ plus cisplatin in HER2-positive gastric cancer (HERBIS-1). Br J Cancer 110: 1163-1168, 2014

19. Ryu MH, Yoo C, Kim JG, Ryoo BY, Park YS, Park SR, Han HS, Chung IJ, Song EK, Lee KH, et al: Multicenter phase II study of trastuzumab in combination with capecitabine and oxaliplatin for advanced gastric cancer. Eur J Cancer 51: 482-488, 2015.

20. Fang FQ: A clinical study of DOF regimenvsSOX regimen as first-line treatment in patients with advanced gastric carcinoma. World Chin J Digestol 22: 4830, 2014

21. Cunningham D, Okines AF and Ashley S: Capecitabine and oxaliplatin for advanced esophagogastric cancer. N Engl J Med 362: 858-859, 2010.

22. Hironaka S, Ueda S, Yasui H, Nishina T, Tsuda M, Tsumura T, Sugimoto N, Shimodaira H, Tokunaga S, Moriwaki T, et al: Randomized, open-label, phase III study comparing irinotecan with paclitaxel in patients with advanced gastric cancer without severe peritoneal metastasis after failure of prior combination chemotherapy using fluoropyrimidine plus platinum: WJOG 4007 trial. J Clin Oncol 31: 4438-4444, 2013.

23. Meads DM, Marshall A, Hulme CT, Dunn JA and Ford HE: The cost effectiveness of docetaxel and active symptom control versus active symptom control alone for refractory oesophagogastric adenocarcinoma: Economic analysis of the COUGAR-02 trial. PharmacoEconomics 34: 33-42, 2016.

24. Ajani JA, D'Amico TA, Almhanna K, Bentrem DJ, Chao J, Das P, Denlinger CS, Fanta P, Farjah F, Fuchs CS, et al: Gastric cancer, version 3.2016, NCCN clinical practice guidelines in oncology. J Natl Compr Cancer Netw 14: 1286-1312, 2016.

25. Nishikawa K, Takahashi T, Takaishi H, Miki A, Noshiro H, Yoshikawa T, Nishida Y, Iwasa S, Miwa H, Masuishi T, et al: Phase II study of the effectiveness and safety of trastuzumab and paclitaxel for taxane- and trastuzumab-naive patients with HER2-positive, previously treated, advanced, or recurrent gastric cancer (JFMC45-1102). Int J Cancer 140: 188-196, 2017.
26. Ford HE, Marshall A, Bridgewater JA, Janowitz T, Coxon FY, Wadsley J, Mansoor W, Fyfe D, Madhusudan S, Middleton GW, et al: Docetaxel versus active symptom control for refractory oesophagogastric adenocarcinoma (COUGAR-02): An open-label, phase 3 randomised controlled trial. Lancet Oncol 15: 78-86, 2014.

27. Thuss-Patience PC, Kretzschmar A, Bichev D, Deist T, Hinke A, Breithaupt K, Dogan Y, Gebauer B, Schumacher G and Reichardt P: Survival advantage for irinotecan versus best supportive care as second-line chemotherapy in gastric cancer-a randomised phase III study of the Arbeitsgemeinschaft Internistische Onkologie (AIO). Eur J Cancer 47: 2306-2314, 2011.

28. Sym SJ, Hong J, Park J, Cho EK, Lee JH, Park YH, Lee WK, Chung M, Kim HS, Park SH and Shin DB: A randomized phase II study of biweekly irinotecan monotherapy or a combination of irinotecan plus 5-fluorouracil/leucovorin (mFOLFIRI) in patients with metastatic gastric adenocarcinoma refractory to or progressive after first-line chemotherapy. Cancer Chemother Pharmacol 71: 481-488, 2013.

29. Maruta F, Ishizone S, Hiraguri M, Fujimori Y, Shimizu F, Kumeda S and Miyagawa S: A clinical study of docetaxel with or without 5'DFUR as a second-line chemotherapy for advanced gastric cancer. Med Oncol 24: 71-75, 2007.

30. Satoh T, Xu RH, Chung HC, Sun GP, Doi T, Xu JM, Tsuji A, Omuro Y, Li J, Wang JW, et al: Lapatinib plus paclitaxel versus paclitaxel alone in the second-line treatment of HER2-amplified advanced gastric cancer in Asian populations: TyTAN-a randomized, phase III study. J Clin Oncol 32: 2039-2049, 2014.

31. Chen SC, Kagedal M, Gao Y, Wang B, Harle-Yge ML, Girish S, Jin $\mathrm{J}$ and Li C: Population pharmacokinetics of trastuzumab emtansine in previously treated patients with HER2-positive advanced gastric cancer (AGC). Cancer Chemother Pharmacol 80: 1147-1159, 2017.

32. Fuchs CS, Tomasek J, Yong CJ, Dumitru F, Passalacqua R, Goswami C, Safran H, Dos Santos LV, Aprile G, Ferry DR, et al: Ramucirumab monotherapy for previously treated advanced gastric or gastro-oesophageal junction adenocarcinoma (REGARD): An international, randomised, multicentre, placebo-controlled, phase 3 trial. Lancet 383: 31-39, 2014.

33. Wilke H, Muro K, Van Cutsem E, Oh SC, Bodoky G, Shimada Y, Hironaka S, Sugimoto N, Lipatov O, Kim TY, et al: Ramucirumab plus paclitaxel versus placebo plus paclitaxel in patients with previously treated advanced gastric or gastro-oesophageal junction adenocarcinoma (RAINBOW): A double-blind, randomised phase 3 trial. Lancet Oncol 15: 1224-1235, 2014.

34. Yuan M, Shi Z, Wang Z, Lv W, Yang Y, Lu F, Zhao Y and Zhong H: The significance of multi-line chemotherapy for advanced gastric cancer: A retrospective analysis. Mol Clin Oncol 6: 606-612, 2017.

35. Zhang W, Yu Y, Fang Y, Wang Y, Cui Y, Shen K and Liu T: Systemic chemotherapy as a main strategy for liver metastases from gastric cancer. Clin Transl Oncol 17: 888-894, 2015.

36. Li J, Qin S, Xu J, Xiong J, Wu C, Bai Y, Liu W, Tong J, Liu Y, $\mathrm{Xu} \mathrm{R}$, et al: Randomized, double-blind, placebo-controlled phase iii trial of apatinib in patients with chemotherapy-refractory advanced or metastatic adenocarcinoma of the stomach or gastroesophageal junction. J Clin Oncol 34: 1448-1454, 2016.

37. Moore KN, Martin LP, O'Malley DM, Matulonis UA, Konner JA, Perez RP, Bauer TM, Ruiz-Soto R and Birrer MJ: Safety and activity of mirvetuximab soravtansine (IMGN853), a folate receptor alpha-targeting antibody-drug conjugate, in platinum-resistant ovarian, fallopian tube, or primary peritoneal cancer: A phase I expansion study. J Clin Oncol 35: 1112-1118, 2017.

38. Yamasaki M, Saito N, Hada Y, Miyamoto S, Okanobu H, Ikeda N, Daido W, Ishiyama S, Deguchi N, Taniwaki M and Ohashi N: Nivolumab therapy for synchronous ALK-positive lung cancer and gastric cancer. Case Rep Oncol 10: 361-367, 2017. 